many
ribosomal
proteins show
dynamic and
heterogeneous
expression
patterns in
the developing
embryo.

\title{
GENE EXPRESSION
}

\section{Personalized ribosomes}

The ribosome has long been thought to act constitutively and to globally regulate protein synthesis during mammalian development. But the sheer complexity of the ribosome, which contains several RNAs and $>70$ ribosomal proteins, may offer an opportunity for more tailored regulation. Indeed, Kondrashov et al. now show that, during mouse development, ribosomal protein L38 (RPL38) controls the translation of a specific set of homeobox (Hox) mRNAs.

There have been previous indications in some organisms that the ribosome may have more regulatory activity, including observations that the loss of some ribosomal proteins can result in tissue-specific defects. When characterizing the mouse mutant tail short, which has been known for more than 50 years

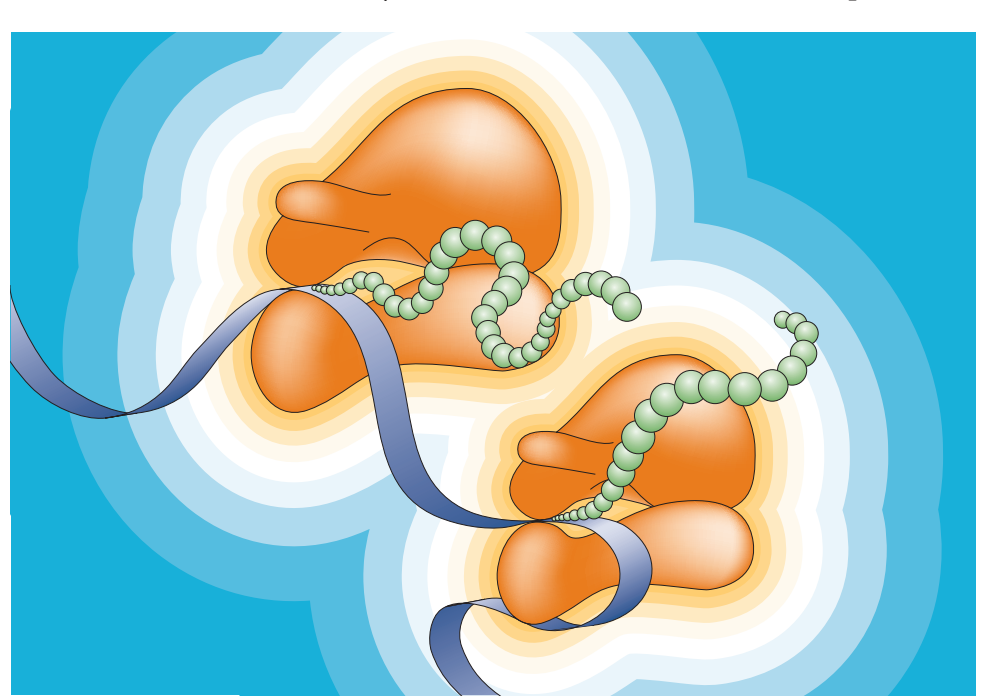

to have impaired skeletal patterning, Kondrashov et al. discovered that the phenotypes of this mouse strain are caused by mutation of Rpl38 specifically. Closer examination revealed that loss of RPL38 disrupted patterning along the axial skeleton.

As Hox genes are important for regulating axial patterning, the authors asked whether these genes might contribute to this phenotype. Initially, this did not seem to be the case, as the expression boundaries of Hox genes were normal. And, using a new transgenic mouse strain that allows protein synthesis to be monitored in vivo, they found that global protein synthesis was also normal in the somites and neural tubes of RPL38 mutants. However, polysome profiling indicated that RPL38 mutants showed decreased protein
(1) So, is this specificity for a subset of mRNAs a peculiarity of RPL38? Probably not, as Kondrashov et al. also found that many ribosomal proteins show dynamic and heterogeneous expression patterns in the developing embryo. Thus, this study lends weight to the idea that ribosome composition and perhaps activity may be adapted in many ways in different cell types, and that this may provide additional specificity during gene expression.

Alison Schuldt

ORIGINAL RESEARCH PAPER Kondrashov, N. et al. Ribosome-mediated specificity in $\mathrm{Hox}$ mRNA translation and vertebrate tissue patterning. Cell 145, 383-397 (2011) 